\title{
PileLine: a toolbox to handle genome position information in next-generation sequencing studies
}

\author{
Daniel Glez-Peña ${ }^{1 *}$, Gonzalo Gómez-López², Miguel Reboiro-Jato', Florentino Fdez-Riverola', David G Pisano²
}

\begin{abstract}
Background: Genomic position (GP) files currently used in next-generation sequencing (NGS) studies are always difficult to manipulate due to their huge size and the lack of appropriate tools to properly manage them. The structure of these flat files is based on representing one line per position that has been covered by at least one aligned read, imposing significant restrictions from a computational performance perspective.

Results: PileLine implements a flexible command-line toolkit providing specific support to the management, filtering, comparison and annotation of GP files produced by NGS experiments. PileLine tools are coded in Java and run on both UNIX (Linux, Mac OS) and Windows platforms. The set of tools comprising PileLine are designed to be memory efficient by performing fast seek on-disk operations over sorted GP files.

Conclusions: Our novel toolbox has been extensively tested taking into consideration performance issues. It is publicly available at http://sourceforge.net/projects/pilelinetools under the GNU LGPL license. Full documentation including common use cases and guided analysis workflows is available at http://sing.ei.uvigo.es/pileline.
\end{abstract}

\section{Background}

Nowadays, commercially available NGS technologies are able to generate in a single run millions of DNA short reads producing a gigabasepair (Gbp) scale throughput at relatively low cost [1]. A crucial step in NGS resequencing workflows is the mapping of DNA short reads against a reference genome in order to obtain text files which represent the sequence information by its genomic position (e.g. .pileup, .bed, .gff, .vcf, etc.). In this context, SAMtools [2] is a well-known open-source package able to manipulate Sequence Alignment Map (SAM) files and, amongst other utilities, its variant caller generates a GP file called pileup able to be further analyzed in NGS-based research studies (e.g. DNA or RNA-seq).

As an example, Ding et al. [3] have characterized novel mutations using data analysis workflows that require a comprehensive GP file handling for variant annotation (i.e. to discard known variants such as SNPs) and case-control comparisons. Nevertheless, the management of this type of files is usually cumbersome and inefficient due to both their large size (about $3 \cdot 10^{9}$ lines

\footnotetext{
* Correspondence: dgpena@uvigo.es

'Higher Technical School of Computer Engineering, University of Vigo,

Ourense, Spain

Full list of author information is available at the end of the article
}

for human genome) and the lack of specific tools able to efficiently manage them from a disk, memory and CPU point of view.

Starting from our experience in giving direct support to wet-lab users requiring NGS analysis we have developed PileLine, a novel and flexible command-line toolbox for efficient management, filtering, comparison and annotation of GP files. The toolbox has been designed to be memory efficient by performing fast seek on-disk operations over sorted GP files. Based on the combination of basic core operations, PileLine provides several functionalities, including $(i)$ full standard annotation with human dbSNP, HGNC Gene Symbol and Ensembl IDs, (ii) custom annotation through standard .bed or .gff files, (iii) two sample (i.e.: case VS control) and $n$ sample comparison at variant level, (iv) generation of SIFT [4], Firestar [5] and PolyPhen [6] compatible outputs for predicting the consequences of non-synonymous coding variants on protein function, and $(v)$ a genotyping quality control (QC) test for estimating performance metrics on detecting homo/heterozygote variants against a given gold standard genotype [7].
C Biomed Central

(c) 2011 Glez-Peña et al; licensee BioMed Central Ltd. This is an Open Access article distributed under the terms of the Creative Commons Attribution License (http://creativecommons.org/licenses/by/2.0), which permits unrestricted use, distribution, and reproduction in any medium, provided the original work is properly cited. 


\section{Implementation}

PileLine is coded in Java and consists of a set of command-line utilities that are easy to integrate in custom workflows or user-friendly frameworks like Galaxy [8]. The tools comprising PileLine are focussed on two different but complementary activities: $(i)$ processing and annotation, implementing simple but reusable operations over input GP files and (ii) analysis, giving support to more advanced and specific requirements (see Table 1 and Additional File 1).

The primary input data of PileLine are GP files (e.g. . pileup from SAMtools) containing the chromosome name and the coordinate position as the two first columns (see Figure 1). The main design principle of the PileLine toolbox is to avoid loading input data into memory, so core functions operate directly on disk. One of the available command-line tools is fastseek, which performs a direct binary search on sorted GP files without requiring an additional index to be created. This functionality provides direct access to any range of genomic coordinates without loading the whole file into memory. Initially, fastseek finds the first and last lines of each sequence and next, performs a binary search on the lines belonging to the queried sequence in order to find the first position within the specified range.

The second design principle of PileLine is focussed on flexibility and modularity. Thus, PileLine tools may be combined with standard UNIX commands allowing custom data analysis workflows. Moreover, the modular design of our toolbox facilitates the inclusion of additional functionalities. With respect to the file formats, while $2 s m c$, nsmc, pileup2sift, pileup2polyphen and pileup2firestar work with specific samtools .pileup file format, fastseek, fastjoin, rfilter, sort and genotest work with generic GP files (i.e.: .pileup, .vcf, .gff, .bed, etc.).

\section{Results and discussion}

PipeLine toolbox contains 10 command-line utilities that have been designed to be memory efficient by performing on-disk operations over sorted GP files. By combining their execution using different arguments and several options the user is able to sketch and execute diverse workflows that can be enhanced by using third party software applications. Here we report several example PileLine applications, further commands and examples of use can be found on the PileLine web site:

a) Case-Control comparisons working with .pileup files: \$ sh YOUR_PATH_TO_PILELINE/cmd/pileline2smc.sh - a <Controll.pileup> - b < Casel. pileup $>-v<$ Controll. variants.pileup $>-w$ $<$ Case1.variants. pileup > - o <youtput 1 . txt>

b) $N$ sample comparisons reporting consistent variants amongst .pileup files:

\$ sh YOUR_PATH_TO_PILELINE/cmd/pilelinensmc.sh -a-samples <Control1.variants. pileup>, <Control2. variants.pileup > , <Control3.variants.pileup $>-$ b-samples < Case1. variants.pileup >,<Case2.variants. pileup >, <Case3.variants.pileup > -o <mycommonvariants_in_Cases.txt>

c) Full annotation of GP files with human dbSNP:

\$ sh YOUR PATH TO PILELINE/cmd/pileline-fastjoin.sh $-\mathrm{a}<\mathrm{GP} f \mathrm{file} \cdot \mathrm{txt}>-\mathrm{b} . / \mathrm{dbSNP} 36.3$. txt -left-outer-join $><$ mydbsNPannotation1.txt>

Table 1 Summary of PileLine functionalities

\begin{tabular}{|c|c|}
\hline Tool & Description \\
\hline \multicolumn{2}{|c|}{ Processing and annotation } \\
\hline fastseek & Retrieves all lines within a specified genome range. \\
\hline fastjoin & Joins two GP input files by genomic coordinate. It can also perform left- and right- outer joins which print orphan lines. \\
\hline rfilter & $\begin{array}{l}\text { Selects only those positions inside at least one of a given set of intervals (.bed or .gff files). It also implements an annotation } \\
\text { mode to report all positions plus an extra column containing all the intervals in which each position is contained. }\end{array}$ \\
\hline sort & Sorts a GP file by genomic coordinate. SAMtools generated pileup files are usually sorted. \\
\hline pileup2sift & Generates a SIFT-compatible change column for each variant line in the GP file. \\
\hline pileup2polyphen & Generates a Polyphen2-compatible change column for each variant line in the GP file. \\
\hline pileup2firestar & Generates a firestar-compatible input for each variant line in the GP file. \\
\hline \multicolumn{2}{|l|}{ Analysis } \\
\hline $2 \mathrm{smc}$ & $\begin{array}{l}\text { Compares two samples (i.e. case VS control) by retrieving all positions where the genotype is discrepant between the two } \\
\text { samples. For each sample a variant GP file is needed, as well as the complete GP file (which includes the invariant positions). }\end{array}$ \\
\hline nsmc & $\begin{array}{l}\text { Compares } n \text { samples of two conditions (i.e. case VS control). Taking one GP file per sample, it reports those samples } \\
\text { containing each position and also performs a Fisher's exact test to find reproducible and characteristic positions. }\end{array}$ \\
\hline genotest & $\begin{array}{l}\text { Performs a QC test on genotyping. Compares two genotypes (experimental VS gold standard) and evaluates the } \\
\text { performance on detecting homo/heterozygous variants. It also generates data to plot a ROC curve in order to estimate the } \\
\text { best SNP quality threshold. See Additional File } 1 \text { for an example output. }\end{array}$ \\
\hline
\end{tabular}




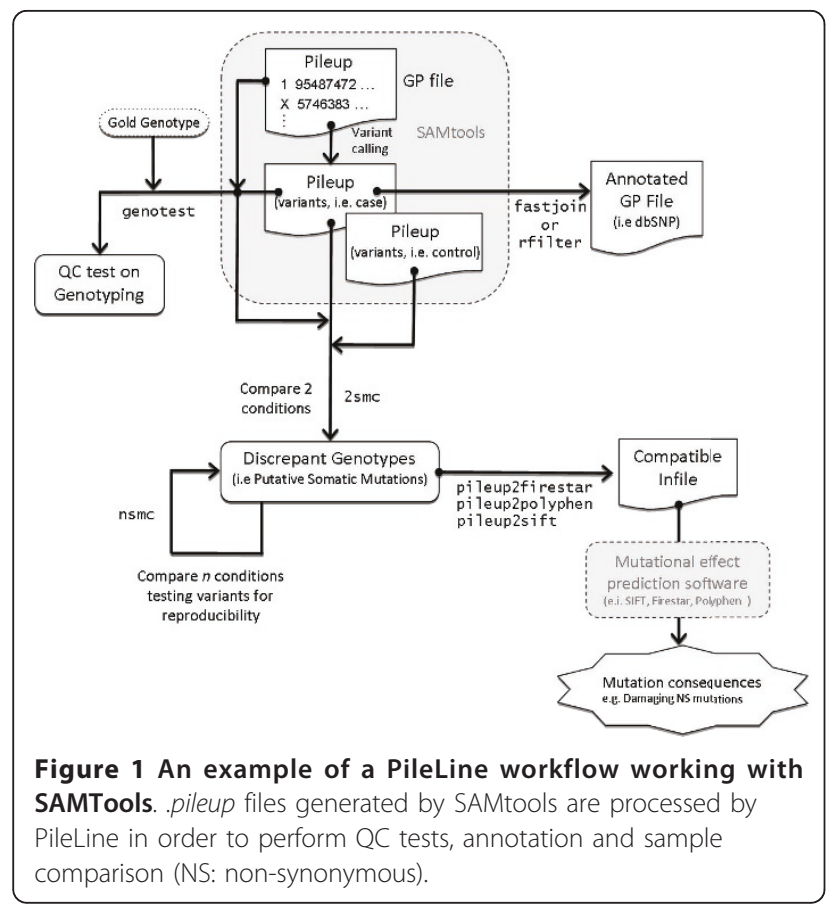

\#HGNC Gene Symbol, Ensembl IDs and custom annotations are also allowed and may be supplied through standard bed or gff files.

d) Generate input for external mutational effects prediction software (i.e. SIFT):

\$ sh YOUR_PATH_TO_PILELINE/cmd/pileline-

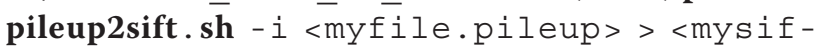
tinput.txt>

\# Polyphen2 and Firestar inputs are also allowed.

e) Print a given range of a GP file (without indexing).

\$ sh YOUR_PATH_TO_PILELINE/cmd/pileline-

fastseek.sh - $\bar{p}<$ GP_file $>$-s chr10:100:10000 $><$ mychrrange.txt $>$

PileLine performs efficiently on a standard PC (Intel Core 2,33 GHz with 1 Gb RAM), where initial tests with the fastseek command showed good performance being able to retrieve 1400 random positions per second on a file of $\sim 174$ millions of lines ( $\sim 5,5 \mathrm{~Gb})$. This behaviour allows, for example, to retrieve all positions from a .pileup file containing known SNPs ( 4 million) in approximately 45 minutes consuming less than $150 \mathrm{Mb}$ of RAM.

Although an optimal search performance could be attained by using auxiliary indexes, this approach requires an additional step for building the supporting files. Moreover, the performance degrades linearly as the input GP file grows in size, and its generation takes a considerable amount of time. PileLine was designed to avoid indexing but, by performing binary searches instead of sequential searches (taking advantage of sorted GP files), it scales reasonably well since its complexity is $\mathrm{O}\left(\log _{2} \mathrm{~N}\right)$, where $\mathrm{N}$ is the number of lines of the input GP file.

The PileLine toolbox is maintained and distributed using a concurrent CVS version control system at SourceForge. The community platform provides wiki support through MediaWiki.

\section{Conclusion}

In this work we have presented PileLine, a toolbox for the efficient processing of standard genomic position files such as .pileup, .bed, .gff and .vcf. Based on the combination of basic core tools, PileLine provides a catalogue of functions to analyze, compare, filter and annotate GP files giving support to common NGS analysis workflows. Given the growing number of NGS-oriented applications, PileLine has been implemented using a modular design to facilitate the inclusion of new functionalities. PileLine toolbox and source code are freely available from http://sourceforge.net/projects/pilelinetools and licensed under the terms of the GNU Lesser General Public License. Examples of use, commands help, guided analysis workflows, example files and full documentation are available in PileLine's wiki at http://sing.ei.uvigo.es/pileline.

\section{Availability and requirements \\ Project name: PileLine}

Project home page: http://sing.ei.uvigo.es/pileline

Operating systems: Windows, Unix-like (Linux, Mac OSX)

Programming language: Java

Other requirements: Java Runtime Environment (JRE) 1.6, Apache Ant 1.7

License: GNU LGPL

\section{Additional material}

Additional file 1: Example output of a genotyping test for quality control. Genotest metrics table description. It may be obtained by using -print-help-table argument.

\section{Acknowledgements}

We thank G. López and JM. Rodríguez for helping with PileLine and Firestar integration. This work has been partially funded by the Spanish Ministry of Science and Innovation, the Plan E from the Spanish Government and the European Union from the ERDF (TIN2009-14057-C03-02). DG-P acknowledges the Angeles Alvariño contract and a visiting scholar grant from Xunta de Galicia (Spain) and the European Social Fund.

\section{Author details}

${ }^{1}$ Higher Technical School of Computer Engineering, University of Vigo, Ourense, Spain. ${ }^{2}$ Bioinformatics Unit (UBio), Structural Biology and Biocomputing Programme, Spanish National Cancer Research Centre (CNIO), Madrid, Spain. 


\section{Authors' contributions}

DG-P programmed the PileLine application. GGL provided use cases, tested the usability of the software and generated PileLine documentation. MRJ tested the performance of PileLine tools. DG-P and GGL wrote the paper while FFR and DGP provided comments and discussion. All authors read and approved the final manuscript.

Received: 4 October 2010 Accepted: 24 January 2011

Published: 24 January 2011

\section{References}

1. Metzker ML: Sequencing technologies-the next generation. Nat. Rev. Genet 2009, 11(1):31-46.

2. Li H, Handsaker B, Wysoker A, Fennell T, Ruan J, Homer N, Marth G, Abecasis G, Durbin R: The Sequence Alignment/Map format and SAMtools. Bioinformatics 2009, 25(16):2078-2079.

3. Ding L, Ellis MJ, Li S, Larson DE, Chen K, Wallis JW, Harris CC, McLellan MD, Fulton RS, Fulton LL, Abbott RM, Hoog J, Dooling DJ, Koboldt DC, Schmidt H, Kalicki J, Zhang Q, Chen L, Lin L, Wendl MC, McMichael JF, Magrini VJ, Cook L, McGrath SD, Vickery TL, Appelbaum E, Deschryver K, Davies S, Guintoli T, Lin L, Crowder R, Tao Y, Snider JE, Smith SM, Dukes AF, Sanderson GE, Pohl CS, Delehaunty KD, Fronick CC, Pape KA, Reed JS, Robinson JS, Hodges JS, Schierding W, Dees ND, Shen D, Locke DP, Wiechert ME, Eldred JM, Peck JB, Oberkfell BJ, Lolofie JT, Du F, Hawkins AE, O'Laughlin MD, Bernard KE, Cunningham M, Elliott G, Mason MD, Thompson DM Jr, Ivanovich JL, Goodfellow PJ, Perou CM, Weinstock GM, Aft R, Watson M, Ley TJ, Wilson RK, Mardis ER: Genome remodelling in a basal-like breast cancer metastasis and xenograft. Nature 2010, 464(7291):999-1005.

4. Kumar P, Henikoff S, Ng PC: Predicting the effects of coding nonsynonymous variants on protein function using the SIFT algorithm. Nat Protoc 2009, 4(7):1073-1081.

5. López G, Valencia A, Tress ML: Firestar-prediction of functionally important residues using structural templates and alignment reliability. Nucleic Acids Res 2007, , 35 Web Server: W573-7.

6. Ramensky V, Bork P, Sunyaev S: Human non-synonymous SNPs: server and survey. Nucleic Acids Res 2002, 30(17):3894-3900

7. Harismendy O, Ng PC, Strausberg RL, Wang X, Stockwell TB, Beeson KY, Schork NJ, Murray SS, Topol EJ, Levy S, Frazer KA: Evaluation of next generation sequencing platforms for population targeted sequencing studies. Genome Biol 2009, 10(3):R32.

8. Blankenberg D, Taylor J, Schenck I, He J, Zhang Y, Ghent M

Veeraraghavan N, Albert I, Miller W, Makova KD, Hardison RC, Nekrutenko A: A framework for collaborative analysis of ENCODE data: making largescale analyses biologist-friendly. Genome Res 2007, 17(6):960-964.

\section{Submit your next manuscript to BioMed Central and take full advantage of:}

- Convenient online submission

- Thorough peer review

- No space constraints or color figure charges

- Immediate publication on acceptance

- Inclusion in PubMed, CAS, Scopus and Google Scholar

- Research which is freely available for redistribution

Submit your manuscript at www.biomedcentral.com/submit 